\title{
Aproximação de Problemas de Valores de Fronteira Singulares usando Subsoluções e Supersoluções
}

P.M. LIMA, Departamento de Matemática, Instituto Superior Técnico, Av. Rovisco Pais, 1049-001 Lisboa, Portugal

A.M. OLIVEIRA, Departamento de Matemática, Faculdade de Ciências e Tecnologia, Universidade Nova de Lisboa, 2825 Monte de Caparica, Portugal.

Resumo. No presente trabalho consideram-se problemas de valores de fronteira para um certo tipo de equações diferenciais ordinárias de segunda ordem, conhecidas como equações generalizadas de Emden-Fowler. Estas equações têm numerosas aplicações, nomeadamente em problemas de mecânica de fluidos. Do ponto de vista matemático, os problemas distinguem-se por as soluções apresentarem singularidades num ou em ambos os extremos do intervalo, o que levanta certas dificuldades na aproximação numérica.

\section{Introdução}

O objetivo deste trabalho é o estudo de equações diferenciais ordinárias do tipo

$$
y^{\prime \prime}(x)=w(x) y(x)^{q}
$$

com

$$
w(x)=-g(x) x^{p}
$$

onde $g(x)$ é uma função contínua e positiva em ]0,1[ e $p$ e $q$ são números reais.

Este tipo de equações diferenciais, habitualmente designadas por equações de Emden-Fowler generalizadas, tem numerosas aplicações práticas, nomeadamente em mecânica dos fluidos e física química e do plasma, entre outras áreas.

Neste artigo vamos considerar $q<-1$ e supor a existência de 2 números reais $m$ e $M$ tais que $0<m=\min _{x \in[0,1]} g(x) \leq M=\max _{x \in[0,1]} g(x)$.

O caso particular $m=M=-C$ também será estudado.

Serão procuradas as soluções positivas de (1.1), em ]0,1[, que satisfaçam cada um dos dois seguintes conjuntos de condições de fronteira:

$$
\begin{gathered}
y^{\prime \prime}(0)=y(1)=0, \\
y(0)=y(1)=0 .
\end{gathered}
$$

${ }^{1}$ plima@math.ist.utl.pt 
No caso (1.1)-(1.3), a função $y(x)$ procurada tem uma singularidade em $x=1 \mathrm{e}$ é uma função de classe $C([0,1]) \cap C^{1}([0,1[)$.

No caso (1.1)-(1.4) esta função tem singularidades nos pontos $x=0$ e $x=1$.

Ainda para (1.1)-(1.3), o caso particular $w(x)=\frac{1}{q} x$ surge na mecânica dos fluidos não newtonianos e tem sido abordado por alguns autores $[11,14]$.

Em [8] e [9] são desenvolvidos alguns métodos numéricos para a aproximação deste problema.

O problema (1.1)-(1.4) tem sido também largamente estudado em $[2,4,5,6,10$, $12,15]$.

Um problema semelhante, com condição de fronteira não homogênea em $x=0$ foi tratado em [3].

No presente trabalho iremos generalizar os métodos referidos nos trabalhos anteriores e aplicá-los no estudo da equação (1.1), com diferentes expressões de $w(x)$. Neste sentido, este trabalho é a continuação de [8] e [9], onde foi considerado apenas o caso de $w(x)=x$ e para as condições de fronteira (1.1)-(1.3).

Numa primeira fase, e para cada um dos dois tipos de problemas, (1.1)-(1.3) e (1.1)-(1.4), serão deduzidas aproximações iniciais, subsoluções ou supersoluções (sec. 2), como ponto de partida para a aplicação de métodos iterativos do tipo de Picard ou de Newton (sec. 3).

Ainda na sec. 2, serão enunciados teoremas que garantem a existência e unicidade de (1.1)-(1.3) e (1.1)-(1.4), sob certas condições.

Os métodos iterativos utilizados na sec. 3 darão origem a problemas lineares, que serão posteriormente discretizados, mediante um tratamento numérico envolvendo diferenças finitas (sec. 4).

Deduzidas as fórmulas desses métodos, proceder-se-á em seguida à análise de alguns exemplos concretos, com o auxílio de um algoritmo implementado em linguagem PASCAL.

Para os casos em que a solução, $y(x)$, for conhecida, será feita uma análise de convergência (sec. 5).

\section{Subsoluções e Supersoluções}

Considere-se o problema (1.1)-(1.3).

Defina-se uma função $h(x) \in C^{2}(] 0,1[) \cap C^{1}([0,1[) \cap C([0,1])$ sujeita às condições

$$
\begin{gathered}
-h^{\prime \prime}(x)+w(x) h(x)^{q} \leq 0 \quad(\text { resp. } \geq 0), \quad \\
h^{\prime}(0) \geq 0 \quad(\text { resp. } \leq 0) ; \quad h(1)=0 .
\end{gathered}
$$

Designa-se esta função como subsolução (resp. supersolução) do problema (1.1)-(1.3)

Iremos procurar subsoluções e supersoluções que satisfaçam as seguintes condições:

$$
\lim _{x \rightarrow 0^{+}} h^{\prime}(x)=0
$$




$$
\begin{gathered}
\lim _{x \rightarrow 1^{-}} h(x)=0, \\
\lim _{x \rightarrow 1^{-}} \frac{h(x)}{y(x)}=\alpha \neq 0,
\end{gathered}
$$

onde a última condição significa que a subsolução (ou supersolução) é assintoticamente equivalente à solução perto da singularidade.

Estas 3 condições podem ser satisfeitas escolhendo $h$ da forma

$$
h(x)=B\left(1-x^{p+2}\right)^{\gamma},
$$

onde $B$ e $\gamma$ são parâmetros a determinar.

Em relação à condição (2.1), ela verifica-se para $p>-1$, quaisquer que sejam $B$ e $\gamma$.

A condição (2.2) verifica-se para $\gamma>0$, quaisquer que sejam $p$ e $B$.

Considerando o desenvolvimento assintótico de $h$ quando $x \rightarrow 0$, pode concluirse que a condição (2.3) é satisfeita se

$$
\gamma=\frac{2}{1-q}
$$

Iniciemos o nosso estudo com o caso concreto $w(x)=C x^{p}$, com $C<0$ e $p>-1$ constantes.

Fazendo um estudo analítico da função $h$, obtemos valores $B_{1}$ e $B_{2}$ tais que, se $B \leq B_{1}, h(x)$ é uma subsolução e se $B \geq B_{2} h(x)$ é uma supersolução.

Quando tomadas num sentido estrito estas duas últimas relações, as funções representadas são designadas por subsoluções estritas e supersoluções estritas.

A Tabela 1 sintetiza os resultados obtidos.

\begin{tabular}{|c|c|c|}
\hline subsoluções & supersoluções & \\
\hline$B_{1}=a(-C)^{\frac{1}{1-q}}$ & $B_{2}=b(-C)^{\frac{1}{1-q}}$ & se $0<\gamma \leq \frac{1}{p+2}$ \\
\hline$B_{1}=b(-C)^{\frac{1}{1-q}}$ & $B_{2}=a(-C)^{\frac{1}{1-q}}$ & se $\frac{1}{p+2} \leq \gamma<1$ \\
\hline
\end{tabular}

Tabela 1 - Coeficientes das subsoluções e supersoluções do problema (1.1)-(1.3), para $w(x)=C x^{p}, C<0, \operatorname{com} a=\left(\frac{1}{(p+2)^{2} \gamma(1-\gamma)}\right)^{\frac{1}{1-q}}$ e $b=\left(\frac{1}{(p+2)(p+1) \gamma}\right)^{\frac{1}{1-q}}$.

No caso particular $\gamma=\frac{1}{p+2}$, ou seja, $q=-2 p-3$, a função $h(x)$, com $B=B_{1}=B_{2}$, é a solução, uma vez que é simultaneamente subsolução e supersolução.

Tem-se assim

$$
y(x)=\left(\frac{-C}{p+1}\right)^{\frac{1}{1-q}}\left(1-x^{p+2}\right)^{\frac{1}{p+2}} .
$$

A generalização deste resultado para o caso $0<m \leq g(x) \leq M$ é quase imediata. 
Os resultados para este caso estão representados na Tabela 2

\begin{tabular}{|c|c|c|}
\hline subsoluções & supersoluções & \\
\hline$B_{1}=a(m)^{\frac{1}{1-q}}$ & $B_{2}=b(M)^{\frac{1}{1-q}}$ & se $0<\gamma<\frac{1}{p+2}$ \\
\hline$B_{1}=b(m)^{\frac{1}{1-q}}$ & $B_{2}=a(M)^{\frac{1}{1-q}}$ & se $\frac{1}{p+2} \leq \gamma<1$ \\
\hline
\end{tabular}

Tabela 2 - Coeficientes das subsoluções e supersoluções do problema (1.1)-(1.3), para $w(x)=-g(x) x^{p} \operatorname{com} 0<m \leq g(x) \leq M$, com $a$ e $b$ definidos como na Tabela 1.

Repare-se que neste caso mais genérico não é possível determinar uma solução exata na forma (2.4), pois nunca é verificada a igualdade $B_{1}=B_{2}$.

É possível, no entanto, garantir a existência e unicidade de tal solução, enunciando o seguinte teorema, adaptado de [1], p.110-117.

Teorema 1 Sejam $h_{1}(x)$ e $h_{2}(x)$, respectivamente, uma subsolução estrita e uma supersolução estrita do problema (1.1)-(1.3) que satisfazem as condições

$$
\begin{aligned}
h_{1}(x)<h_{2}(x), & \forall x \in] 0,1[ \\
h_{1}^{\prime}(0) \geq h_{2}^{\prime}(0) \quad \text { e } & h_{1}(1)=h_{2}(1)=0 .
\end{aligned}
$$

Se existirem funções $\Lambda_{1}(x, y)$ e $\Lambda_{2}(x, y)$ tais que

$$
\left.\Lambda_{1}\left(x, y_{1}-y_{2}\right) \leq w(x)\left(y_{1}^{q}-y_{2}^{q}\right) \leq \Lambda_{2}\left(x, y_{1}-y_{2}\right), \quad \forall x \in\right] 0,1[,
$$

com $w(x)$ definido em (1.2), e onde $y_{i}(x)$ satisfaz $h_{1}(x)<y_{i}(x)<h_{2}(x), \forall x \in$ $\left[0,1\left[, i=1,2\right.\right.$, então existe pelo menos uma solução $y(x)$ tal que $h_{1}(x)<y(x)<$ $h_{2}(x)$.

Repare-se que todas as subsoluções do tipo $h_{1}(x)=B_{1}\left(1-x^{p+2}\right)^{\gamma}$ e supersoluções do tipo $h_{2}(x)=B_{2}\left(1-x^{p+2}\right)^{\gamma}$, com os parâmetros $B_{1}$ e $B_{2}$ atrás definidos, satisfazem às condições do Teorema 1. De fato, tem-se $\left.h_{1}(x)<h_{2}(x) \forall x \in\right] 0,1[$, pois $B_{1}<B_{2}$, assim como $h_{1}^{\prime}(0)=h_{2}^{\prime}(0)=0$ e $h_{1}(1)=h_{2}(1)=0$.

Por outro lado, se fizermos $\Lambda_{1}(x, y)=q w(x) h_{2}(x)^{q-1} y$ e $\Lambda_{2}(x, y)=q w(x) h_{1}(x)^{q-1} y$, verificamos facilmente que (2.7) é satisfeita, mediante a aplicação do Teorema de Lagrange. Além disso, se tivermos $p>-1$ e $q<-1$, o Teorema 7.5 de [16] garante-nos a unicidade de tal solução.

Considere-se agora o problema (1.1)-(1.4).

Vamos definir uma função $h(x) \in C^{2}(] 0,1[) \cap C([0,1])$ que satisfaça as condições

$$
\begin{gathered}
\left.-h^{\prime \prime}(x)+w(x) h(x)^{q} \leq 0(\text { resp. } \geq 0), \quad x \in\right] 0,1[ \\
h(0)=0 ; \quad h(1)=0 .
\end{gathered}
$$

Designa-se esta função como subsolução (resp.supersolução) deste problema.

As condições que as subsoluções e supersoluções deverão satisfazer serão para este caso 


$$
\begin{gathered}
\lim _{x \rightarrow 0^{+}} h(x)=0, \\
\lim _{x \rightarrow 1^{-}} h(x)=0, \\
\lim _{x \rightarrow 0+} \frac{h(x)}{y(x)}=\alpha_{1} \neq 0, \\
\lim _{x \rightarrow 1^{-}} \frac{h(x)}{y(x)}=\alpha_{2} \neq 0,
\end{gathered}
$$

onde as duas últimas condições significam que a subsolução (ou supersolução) é assintoticamente equivalente à solução perto das singularidades. Estas 4 condições podem ser satisfeitas escolhendo $h$ da forma

$$
h(x)=B x^{\gamma_{1}}(1-x)^{\gamma_{2}},
$$

onde $B, \gamma_{1}$ e $\gamma_{2}$ são parâmetros que se podem determinar.

Com efeito, se considerarmos os desenvolvimentos assintóticos de $h$ quando $x \rightarrow 0$ e $x \rightarrow 1$, pode, facilmente, concluir-se que as condições (2.9)-(2.12) se verificam se tivermos

$$
\gamma_{1}=\frac{p+2}{1-q}
$$

e

$$
\gamma_{2}=\frac{2}{1-q} .
$$

Tal como no problema (1.1)-(1.3), vamos iniciar o estudo com o caso particular $w(x)=C x^{p}$, com $C<0$ e $p>-2$ constantes.

Um estudo analítico mais detalhado permite-nos determinar valores $B_{1}$ e $B_{2}$ para os quais se $B \leq B_{1}, h(x)$ representa uma subsolução e se $B \geq B_{2}, \quad h(x)$ representa uma supersolução.

Podemos sintetizar na Tabela 3 os resultados obtidos, que são válidos para $\gamma_{1}<1$ e $\gamma_{2}<1$.

\begin{tabular}{|c|c|c|}
\hline subsoluções & supersoluções & \\
\hline$B_{1}=a(-C)^{\frac{1}{1-q}}$ & $B_{2}=b(-C)^{\frac{1}{1-q}}$ & se $\gamma_{1}+\gamma_{2} \leq 1$ \\
\hline$B_{1}=b(-C)^{\frac{1}{1-q}}$ & $B_{2}=a(-C)^{\frac{1}{1-q}}$ & se $\gamma_{1}+\gamma_{2}>1$ \\
\hline
\end{tabular}

Tabela 3 - Coeficientes das subsoluções e supersoluções do problema (1.1)-(1.4), para $w(x)=C x^{p}, C<0, \operatorname{com} a=\left(\frac{1}{\gamma_{M}\left(1-\gamma_{M}\right)}\right)^{\frac{1}{1-q}}, b=\left(\frac{\gamma_{1}+\gamma_{2}}{\gamma_{1} \gamma_{2}}\right)^{\frac{1}{1-q}}$ e $\gamma_{M}=$ $\max \left(\gamma_{1}, \gamma_{2}\right)$.

Quando $\gamma_{1}+\gamma_{2}=1$, ou seja, quando $q=-p-3$, tem-se $B=B_{1}=B_{2}=$ $=\left(\frac{-C}{\gamma_{1} \gamma_{2}}\right)^{\frac{1}{1-q}}$, pelo que é obtida a expressão analítica da solução 


$$
y(x)=\left(\frac{-C}{\gamma_{1} \gamma_{2}}\right)^{\frac{1}{1-q}} x^{\gamma_{1}}(1-x)^{\gamma_{2}} .
$$

Estendam-se estes resultados para uma função mais genérica, com $g(x) \in[m, M]$. A síntese dos resultados está representada na Tabela 4.

\begin{tabular}{|c|c|c|}
\hline subsoluções & supersoluções & \\
\hline$B_{1}=a(m)^{\frac{1}{1-q}}$ & $B_{2}=b(M)^{\frac{1}{1-q}}$ & se $\gamma_{1}+\gamma_{2} \leq 1$ \\
\hline$B_{1}=b(m)^{\frac{1}{1-q}}$ & $B_{2}=a(M)^{\frac{1}{1-q}}$ & se $\gamma_{1}+\gamma_{2}>1$ \\
\hline
\end{tabular}

Tabela 4 - Coeficientes das subsoluções e supersoluções do problema (1.1)-(1.4), para $w(x)=-g(x) x^{p}$ com $0<m \leq g(x) \leq M$, com $a$ e $b$ definidos como na Tabela 3.

Neste caso não é possível determinar uma solução do tipo (2.13), uma vez que se tem sempre $B_{1} \neq B_{2}$.

No entanto, a existência de uma solução é garantida mediante o Teorema 2, que é uma adaptação ao nosso caso, do Teorema 1 de [7].

Teorema 2 Sejam $h_{1}(x)$ e $h_{2}(x)$, respectivamente uma subsolução e uma supersolução do problema $(1.1)-(1.4)$. Se existir uma função $j(x) \in C(] 0,1[)$ tal que

$$
\left|g(x) x^{p} y^{q}\right| \leq j(x)
$$

para todo par $(x, y)$ pertencente ao conjunto

$$
D=\left\{(x, y) \in R^{2}: 0<x<1, h_{1}(x) \leq y \leq h_{2}(x)\right\}
$$

e verifica

$$
\int_{0}^{1} t(1-t) j(t) d t<+\infty
$$

então existe pelo menos uma solução $y(x)$ do problema $(1.1)-(1.4)$, que satisfaz $h_{1}(x) \leq y(x)<h_{2}(x)$.

De fato, basta escolher a função $j(x)=x^{p} g(x) h_{1}(x)^{q} \operatorname{com} h_{1}(x)$ na forma $(2.13)$ e $B$ de acordo com as Tabelas 3 ou 4 . Neste caso, uma vez que $q<0$, temos $h_{1}(x)^{q}>y^{q}, \forall(x, y) \in D$, donde resulta $(2.17)$.

Por outro lado, com $h_{1}(x)$ na forma (2.13), a integral em (2.18) toma a forma

$$
B^{q} \int_{0}^{1} t^{\gamma_{1} q+p+1}(1-t)^{\gamma_{2} q+1} g(t) d t
$$

e esta integral só é finita se os expoentes que aparecem na função integranda forem ambos maiores que -1 . Isso decorre imediatamente de (2.14) e (2.15), para $p>-2$.

Finalmente, a unicidade da solução resulta, de acordo com o Teorema 2 de [16], do fato de a função $-w(x) y(x)^{q}$ ser uma fun ção decrescente em $y$, para cada $x \in] 0,1[$. 


\section{Métodos Iterativos}

Iremos agora abordar dois métodos iterativos, o de Picard e o de Newton, que nos permitirão transformar o problema não linear (1.1) numa sequência de problemas lineares

Estes métodos, cujas aproximações iniciais, subsoluções e supersoluções foram estabelecidas no capítulo anterior, foram estudados em [13], [8] e [9].

Podemos reescrever a equação (1.1) na forma

$$
y^{\prime \prime}(x)=\beta f(x, y)
$$

com

$$
\beta=-1
$$

e

$$
f(x, y)=g(x) x^{p} y(x)^{q} .
$$

As iteradas de Newton tomam então a forma

$$
y_{j+1}^{\prime \prime}(x)=-\left(g(x) x^{p} y_{j}^{q}(x)+q g(x) x^{p} y_{j}^{q-1}(x)\left(y_{j+1}(x)-y_{j}(x)\right)\right),
$$

ou seja,

$$
y_{j+1}^{\prime \prime}(x)+q g(x) x^{p} y_{j}^{q-1}(x) y_{j+1}(x)=(q-1)\left(g(x) x^{p} y_{j}^{q}(x)\right)
$$

com

$$
y_{j+1}^{\prime}(0)=y_{j+1}(1)=0, \quad j=0,1, \ldots
$$

para as condições de fronteira (1.3), e com

$$
y_{j+1}(0)=y_{j+1}(1)=0, \quad j=0,1, \ldots
$$

para as condições de fronteira (1.4).

As iteradas de Picard são dadas pela fórmula

$$
y_{j+1}^{\prime \prime}(x)-\mu^{2}(x) y_{j+1}(x)=-\left(g(x) x^{p} y_{j}^{q}(x)+\mu^{2}(x) y_{j}(x)\right),
$$

onde a função $\mu^{2}$ deve satisfazer a condição

$$
\left.\mu^{2}(x) \geq-\frac{\partial f(x, y)}{\partial y}, \quad \forall x \in\right] 0,1[,
$$

de modo a garantir a monotonia das iteradas (ver [13]). No nosso caso, a condição (3.9) será satisfeita se tivermos

$$
\left.\mu^{2}(x)=-q g(x) x^{p} h_{1}^{q-1}(x), \quad \forall x \in\right] 0,1[,
$$

onde $h_{1}$ é uma subsolução estrita da equação não linear.

Substituindo (3.10) em (3.8), obtém-se então 


$$
y_{j+1}^{\prime \prime}(x)+q g(x) x^{p} h_{1}^{q-1}(x) y_{j+1}(x)=\left(q h_{1}^{q-1}(x)-y_{j}^{q-1}(x)\right) g(x) x^{p} y_{j}(x)
$$

com as condições (3.6) e (3.7), respectivamente, no caso das condições de fronteira (1.3) e (1.4). A convergência destes dois métodos é garantida pelos teoremas que se seguem e cujas demonstrações iremos omitir.

Teorema 3 Seja $y_{0}(x)=h_{1}(x)$ uma subsolução do problema (1.1)-(1.3) (resp.(1.1)(1.4)). Então, a sucessão de iteradas (3.5) - (3.6) (resp.(3.5) - (3.7)) é monótona crescente e converge uniformemente para a solução.

Teorema 4 Seja $y_{0}(x)=h_{1}(x)$ uma subsolução do problema (1.1)-(1.3) (resp.(1.1)(1.4)). Então, a sucessão de iteradas (3.8) - (3.6) (resp.(3.8) - (3.7)) é monótona crescente e converge uniformemente para a solução.

Teorema 5 Seja $y_{0}(x)=h_{2}(x)$ uma supersolução do problema (1.1)-(1.3) (resp.(1.1)(1.4)). Então, a sucessão de iteradas (3.8) - (3.6) (resp.(3.8) - (3.7)) é monótona decrescente e converge uniformemente para a solução.

\section{Métodos de Discretização}

Iremos utilizar um esquema de diferenças finitas com o propósito de discretizar as equações (3.5) e (3.8).

Seja $h$ o passo da discretização e considere-se uma rede $\left\{x_{i}\right\}_{i=0,1,2, \ldots, N}$, com $x_{i}=i h$. Aproximando a $2^{a}$ derivada por uma diferença central de segunda ordem, tem-se

$$
y_{j+1}^{\prime \prime}\left(x_{i}\right) \approx \frac{y_{j+1}\left(x_{i+1}\right)-2 y_{j+1}\left(x_{i}\right)+y_{j+1}\left(x_{i-1}\right)}{h^{2}} .
$$

Por outro lado, as condições (3.6) e (3.7) dão lugar, respectivamente, a

$$
y_{j+1}\left(x_{1}\right) \approx y\left(x_{0}\right) ; \quad y_{j+1}\left(x_{N}\right)=0
$$

e

$$
y_{j+1}\left(x_{0}\right)=0 ; \quad y_{j+1}\left(x_{N}\right)=0 .
$$

Escrevendo a equação (3.5) para $x=x_{i}, i=1, \ldots, N-1$, e aproximando a $2^{a}$ derivada de acordo com (4.1), obtêm-se as igualdades

$$
\begin{aligned}
\left(\frac{1}{h^{2}}\right) y_{j+1, h}\left(x_{i+1}\right) & +\left(q x_{i}^{p} g\left(x_{i}\right) y_{j, h}^{q-1}\left(x_{i}\right)-\frac{2}{h^{2}}\right) y_{j+1, h}\left(x_{i}\right)+\left(\frac{2}{h^{2}}\right) y_{j+1, h}\left(x_{i-1}\right)= \\
& =g\left(x_{i}\right) x_{i}^{q} y_{j, h}^{q}\left(x_{i}\right)(q-1), \quad i=1, . ., N-1,
\end{aligned}
$$

onde $y_{j+1, h}\left(x_{i}\right)$ representa um valor aproximado de $y_{j+1}\left(x_{i}\right)$. Completando com as condições (4.2), no caso do problema (1.1)-(1.3) ou com as condições (4.3), no 
caso do problema (1.1)-(1.4), obtém-se um sistema linear de $N+1$ equações nas incógnitas $y_{j+1, h}\left(x_{i}\right)$.

Por outro lado, a discretização das equações (3.11) dá origem ao sistema

$$
\begin{gathered}
\left(\frac{1}{h^{2}}\right) y_{j+1, h}\left(x_{i+1}\right)+\left(q x_{i}^{p} g\left(x_{i}\right) h_{1}^{q-1}\left(x_{i}\right)-\frac{2}{h^{2}}\right) y_{j+1, h}\left(x_{i}\right)+\left(\frac{1}{h^{2}}\right) y_{j+1, h}\left(x_{i-1}\right)= \\
=\left(q h_{1}^{q-1}\left(x_{i}\right)-y_{j, h}^{q-1}\left(x_{i}\right)\right) g\left(x_{i}\right) x_{i}^{p} y_{j, h}\left(x_{i}\right), \quad i=1, . ., N-1,
\end{gathered}
$$

que se completa também com as condições (4.2), no caso do problema (1.1)-(1.3), ou com as condições (4.3), no caso do problema (1.1)-(1.4).

Deste modo, em qualquer dos casos obtém-se um sistema linear cuja matriz é tridiagonal e que é resolvido pelos processos usuais.

\section{Exemplos Numéricos e Análise de Convergência}

A partir da implementação de um algoritmo em linguagem PASCAL, foram testados alguns exemplos relativos aos problemas (1.1)-(1.3) e (1.1)-(1.4).

$\mathrm{O}$ critério de parada para cada um dos dois métodos iterativos foi

$$
\left\|\mathbf{y}_{j+1, h}-\mathbf{y}_{j, h}\right\|_{2}<10^{-6}
$$

onde $\mathbf{y}_{j, h}$ e $\mathbf{y}_{j+1, h}$ são aproximações de iteradas consecutivas. Foram utilizados diferentes passos de discretização consoante os exemplos estudados.

Nas Figuras 1 e 2, estão representados os gráficos das soluções exatas, no caso das condições de fronteira (1.3) e (1.4), respectivamente, com $p=-0.5,0,0.5$ e $g(x)=-C=1$. Estas soluções foram obtidas pelas fórmulas (2.6) e (2.16). As soluções aproximadas correspondentes, obtidas pelo método proposto, quando representadas no mesmo gráfico confundem-se com as exactas(ver gráfico do erro relativo na Figuras 5 e 6).

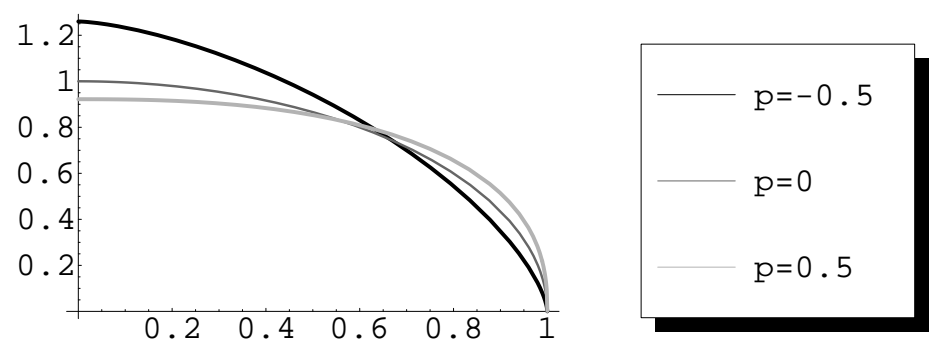

Figura 1: Soluções exatas no caso das condições de fronteira (3) com diferentes valores de $p, q=-2 p-3$ e $g(x)=-C=1$. 

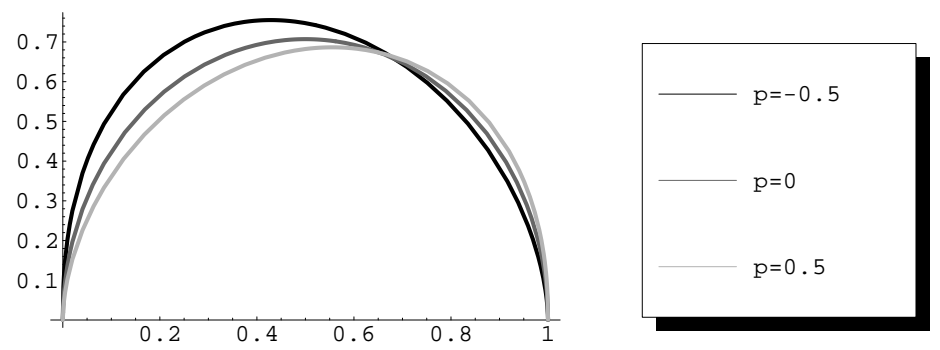

Figura 2: Soluções exatas no caso das condições de fronteira (4) com diferentes valores de $p, q=-p-3$ e $g(x)=-C=1$.

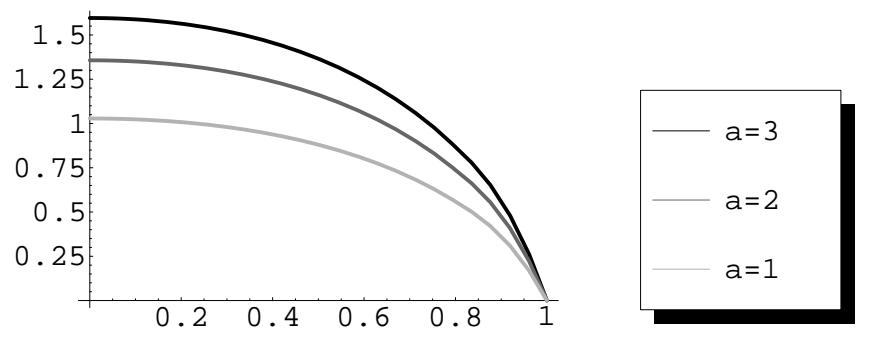

Figura 3: Soluções aproximadas no caso das condições de fronteira (3) para $p=0$, $q=-1.5$ e $g(x)=a e^{x}$, com diferentes valores de $a$.

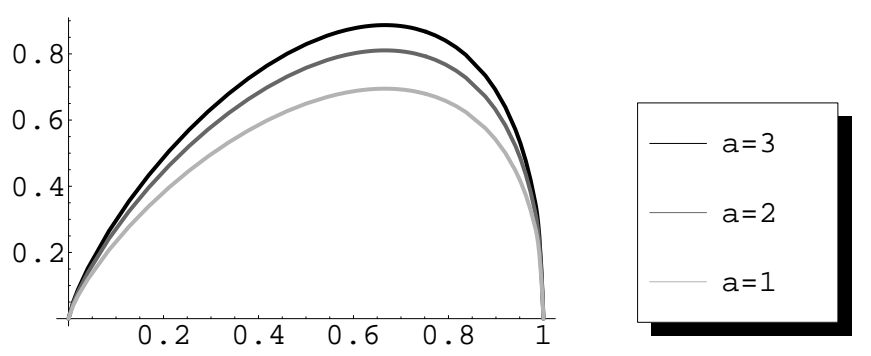

Figura 4: Soluções aproximadas no caso das condições de fronteira (4) para $p=$ $1.5, q=-3.5$ e $g(x)=a e^{x}$, com diferentes valores de $a$. 
Na Figura 3, está representado o gráfico da solução aproximada no caso das condições de fronteira (1.3) para $p=0, q=-1.5$ e $g(x)=a e^{x}$, com $a=1,2,3$.

Na Figura 4, está representado o gráfico da solução aproximada no caso das condições de fronteira (1.4) para $p=1.5, q=-3.5 \mathrm{e}$

$g(x)=a e^{x}, \operatorname{com} a=1,2,3$.

Os gráficos destas duas últimas figuras foram traçados com base nos resultados numéricos obtidos através do nosso programa, tomando como aproximações iniciais subsoluções ou supersoluções es do tipo atrás considerado, e com $h=\frac{1}{1000}$. Notese que, conforme foi assinalado na sec. 2, nestes casos não é possível determinar analiticamente a solução.

Para efeitos de análise de convergência, foram realizados testes numéricos para 5 casos distintos em que a solução exata é conhecida.

Caso 1. Neste caso usam-se as condições de fronteira (1.3) e os valores dos parâmetros são $p=0.5, q=-4.0$ e $g(x)=-C=0.25$.

Tomou-se como estimativa da ordem de convergência o valor $k$ definido por

$$
k=\frac{\log _{2}\left(\frac{E_{2}}{E_{1}}\right)}{\log _{2}\left(\frac{h_{2}}{h_{1}}\right)},
$$

onde $E_{i}$ é o erro relativo calculado com passo $h_{i}, i=1,2$. Neste caso tem-se $k \approx 1.02$.

Caso 2. Neste caso usam-se as condições de fronteira (1.3) e os valores dos parâmetros são $p=-0.5, q=-2.0$ e $g(x)=-C=0.50$. O valor aproximado de $k$ é 0.53 .

Caso 3. Neste caso usam-se as condições de fronteira (1.4) e os valores dos parâmetros são $p=0, q=-3.0$ e $g(x)=-C=1.00$. O valor aproximado de $k$ é 1.0.

Caso 4. Neste caso usam-se as condições de fronteira (1.4) e os valores dos parâmetros são $p=-0.5, q=-2.5$ e $g(x)=-C=1.00$. O valor aproximado de $k$ é 0.9 .

Caso 5. Neste caso usam-se as condições de fronteira (1.4) e os valores dos parâmetros são $p=-1.0, q=-2.0$ e $g(x)=-C=1.00$. O valor aproximado de $k$ é 0.7 .

Em qualquer dos casos, a estimativa da ordem de convergência acima referida é válida para valores de $x$ suficientemente distantes das singularidades.

Nas Figuras 5 e 6, estão representados os gráficos do comportamento do erro relativo como função de $x \operatorname{com} h=\frac{1}{100}$.

Nas Figuras 7 e 8, estão representados os gráficos do comportamento do logaritmo (de base 2) do erro relativo como função do logaritmo (de base 2) de $h$, para valores de $h$ compreendidos entre $\frac{1}{800}$ e $\frac{1}{100}$, considerando o ponto $x=0$, no caso (1.3), e o ponto $x=0.5$, no caso (1.4).

Para traçar os gráficos, recorremos a um programa em MATHEMATICA, que permitiu interpolar os resultados numéricos obtidos. 


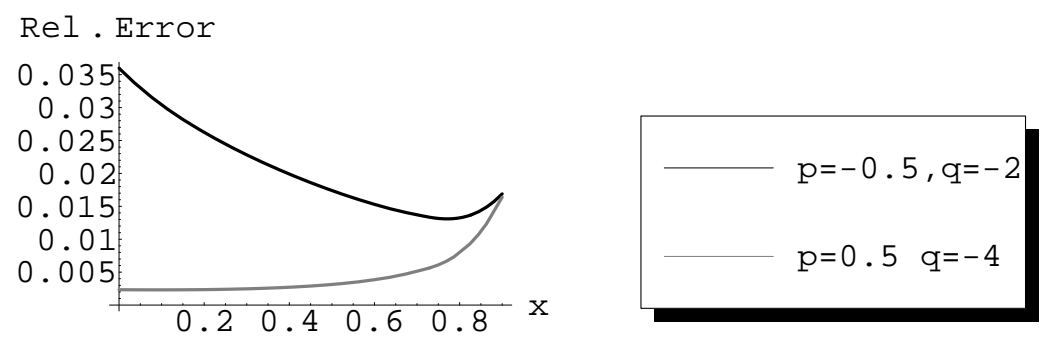

Figura 5: Erro relativo no caso das condições de fronteira (3) em função de $x$, no caso de $h=1 / 100$.

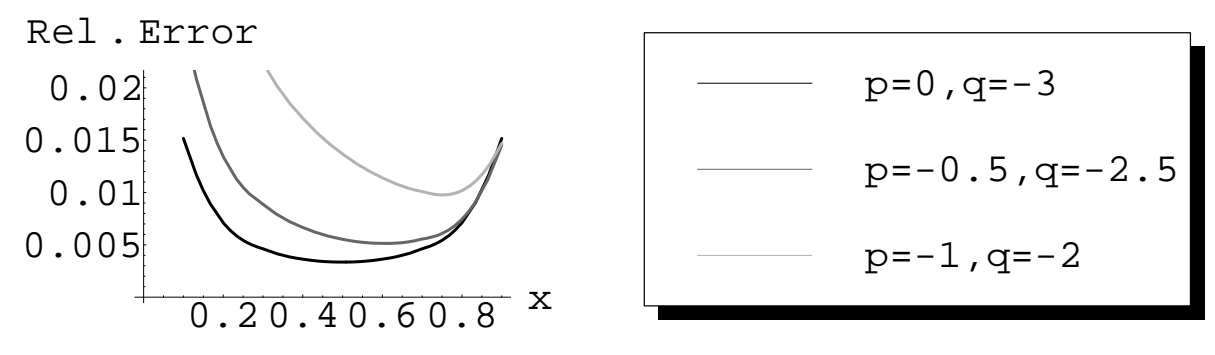

Figura 6: Erro relativo no caso das condições de fronteira (4) como função de $x$, no caso de $h=1 / 100$.

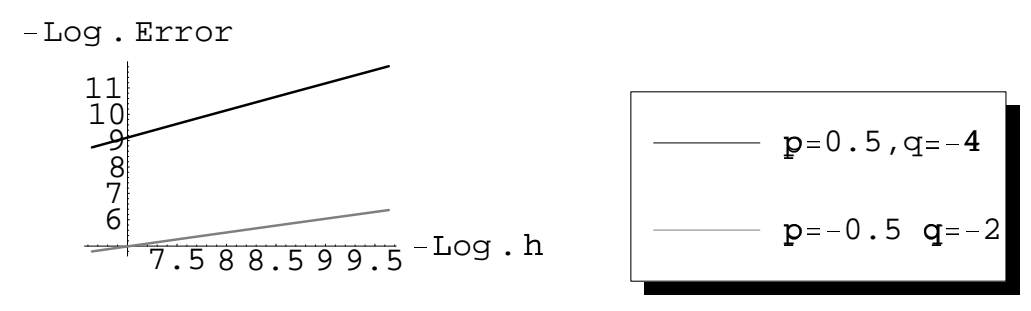

Figura 7: Log. do erro relativo no caso das condições de fronteira (3) como função do Log. de $h$ 


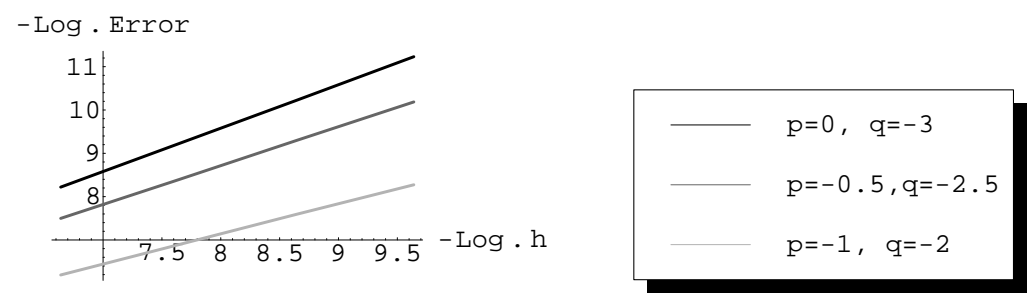

Figura 8: Log. do erro relativo no caso das condições de fronteira (4) como função do Log. de $h$.

\section{Conclusões}

Note-se que apesar de usarmos diferenças finitas de $2^{a}$ ordem, a ordem de convergência esperada neste caso é inferior, devido às singularidades da solução. Não estando ainda concluída a análise teórica da convergência para este caso, podemos apenas conjecturar, com base nos resultados obtidos, sobre a ordem de convergência.

Assim, em todos os exemplos considerados, verifica-se que a estimativa da ordem de convergência é próxima de 1 ou inferior a 1 , dependendo sobretudo dos valores de $p$. Mais precisamente, para valores de $p$ não negativos obtêm-se valores de $k$ próximos de 1 , os quais se tornam mais baixos à medida que $p$ diminui.

Os testes realizados mostraram também que o erro relativo dos resultados e, por conseguinte, a sua ordem de convergência, praticamente não dependem da constante $a$, no caso de $g(x)=a e^{x}$, para ambos os tipos de condições de fronteira (estes valores mantêm-se constantes, pelo menos, até à sexta casa decimal, quando $a$ varia de 1 a 3). Tal está de acordo com as previsões teóricas, já que o valor de $a$ não tem influência no carácter da singularidade.

Uma forma de diminuir o efeito da singularidade e acelerar a convergência será utilizar uma substituição de variável que permita obter uma solução mais regular junto dos extremos. Essa abordagem foi aplicada, por exemplo, em [9], tendo conduzido a métodos numéricos com uma ordem de convergência superior. Num trabalho futuro, os autores tencionam analisar as substituições de variável aplicáveis aos casos aqui estudados e adaptar os algoritmos numéricos ao uso dessa técnica.

\section{Referências}

[1] P. Bailey, L. Shampine, P. Waltman, "Nonlinear Two-point Boundary-value Problems", Academic Press, New York, 1968.

[2] J.V. Baxley, Some singular nonlinear boundary value problems, SIAM J. Math. Anal., 22 (1991), 463-479. 
[3] M. Carpentier and P. Lima, Numerical solution of a sublinear singular boundary-value problem, in Proceedings of the International Conference on Enhancement and Promotion of Computational Methods in Engineering and Science, Macau, 1999.

[4] A. Fink, J. Gatica, G.E. Hernandez, P. Waltman, Approximation of solutions of singular second-order boundary value problems, SIAM J. Math. Anal., 22 (1991), 440-462.

[5] R.C. Flagg, C.D. Luning and W.L. Perry, Implementation of new iterative techniques for solution of Thomas-Fermi and Emden-Fowler equations, $J$. Comp.Phys., 38 (1980), 396-405.

[6] J. Gatica, V. Oliker, P. Waltman, Singular nonlinear boundary value problems for second-order ordinary differential equations, J. Diff. Eq., 79 (1989), 62-78.

[7] P. Habets and F. Zanolin, Upper and lower solutions for a generalized EmdenFowler equation, J. Math. Anal. Appl., 181 (1994), 684-700.

[8] P.M. Lima and M.P. Carpentier, Iterative methods for a singular boundaryvalue problem, J. Comp. Appl. Math., , 111 (1999), 173-186.

[9] P.M. Lima and M.P. Carpentier, Numerical solution of a singular boundaryvalue problem in non-newtonian fluid mechanics, Comp. Phys. Comm., 126 (2000,) 114-120.

[10] C.D. Luning and W.L. Perry, Positive solutions of negative exponent generalized Emden-Fowler BVP, SIAM J. Math. Anal., 12 (1981), 874-879.

[11] C.D. Luning and W.L. Perry, An iterative method for solution of a boundary value problem in non-newtonian fluid flow, J. Non-Newt. Fl. Mech., 15 (1984), 145-154.

[12] C.D. Luning and W.L. Perry, Iterative solution of negative exponent EmdenFowler problems, Internat. J. Math. Math. Sci., 13(1990), 159-164.

[13] J.W. Mooney, A unified approach to the solution of certain classes of nonlinear boundary value problems using monotone iterations, Nonl. Anal., 3 (1979), 449-465.

[14] A. Nachman and A. Callegari, A nonlinear singular boundary- value problem in the theory of pseudoplastic fluids, SIAM J. Appl.Math., 38 (1980), 275-281

[15] S. Taliaferro, A nonlinear singular boundary value problem, Nonl. Anal., 3 (1979), 897-904.

[16] A. Tineo, A comparison theorem for second order ODE's and applications to singular problems, J. Diff. Eq., 116 (1995), 16-30.

[17] J.W. Wong, On the Generalized Emden-Fowler Equation, SIAM Review, 17 (1975), 339-360. 